\title{
Identification of key candidate genes involved in melanoma metastasis
}

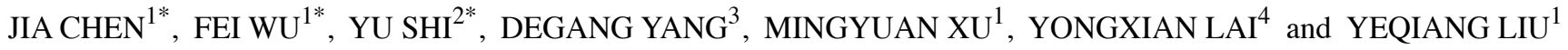 \\ Departments of ${ }^{1}$ Dermatopathology, ${ }^{2}$ Medical Cosmetology, ${ }^{3}$ Treatment and ${ }^{4}$ Dermatologic Surgery, \\ Tongji University Affiliated Shanghai Skin Disease Hospital, Shanghai 200443, P.R. China
}

Received May 10, 2018; Accepted January 18, 2019

DOI: $10.3892 / \mathrm{mmr} .2019 .10314$

\begin{abstract}
Metastasis is the most lethal stage of cancer progression. The present study aimed to investigate the underlying molecular mechanisms of melanoma metastasis using bioinformatics. Using the microarray dataset GSE8401 from the Gene Expression Omnibus database, which included 52 biopsy specimens from patients with melanoma metastasis and 31 biopsy specimens from patients with primary melanoma, differentially expressed genes (DEGs) were identified, subsequent to data preprocessing with the affy package, followed by Gene Ontology and Kyoto Encyclopedia of Genes and Genomes (KEGG) pathway enrichment analyses. A protein-protein interaction (PPI) network was constructed. Mutated genes were analyzed with 80 mutated cases with melanoma from The Cancer Genome Atlas. The overall survival of key candidate DEGs, which were within a filtering of degree $>30$ criteria in the PPI network and involved three or more KEGG signaling pathways, and genes with a high mutation frequency were delineated. The expression analysis of key candidate DEGs, mutant genes and their associated genes were performed on UALCAN. Of the 1,187 DEGs obtained, 505 were upregulated and 682 were downregulated. 'Extracellular exosome' processes, the 'amoebiasis' pathway, the 'ECM-receptor interaction' pathway and the 'focal adhesion' signaling pathway were significantly enriched and identified as important processes or signaling pathways. The overall survival analysis of
\end{abstract}

Correspondence to: Dr Yongxian Lai, Department of Dermatologic Surgery, Tongji University Affiliated Shanghai Skin Disease Hospital, 1278 Baode Road, Shanghai 200443, P.R. China E-mail:michael_lai@126.com

Correspondence to: Dr Yeqiang Liu, Department of Dermatopathology, Tongji University Affiliated Shanghai Skin Disease Hospital, 1278 Baode Road, Shanghai 200443, P.R. China

E-mail: xiaoshui0_0@126.com

*Contributed equally

Key words: melanoma, metastasis, differentially expressed gene, pathway, overall survival phosphoinositide-3-kinase regulator subunit 3 (PIK3R3), centromere protein $\mathrm{M}(C E N P M)$, aurora kinase A (AURKA), laminin subunit $\alpha 1$ (LAMA1), proliferating cell nuclear antigen (PCNA), adenylate cyclase 1 ( $A D C Y 1)$, BUB1 mitotic checkpoint serine/threonine kinase $(B U B 1)$, NDC80 kinetochore complex component $(N D C 80)$ and protein kinase $\mathrm{C} \alpha(P R K C A)$ in DEGs was statistically significant. Mutation gene analysis identified that BRCA1-associated protein $1(B A P 1)$ had a higher mutation frequency and survival analysis, and its associated genes in the $B A P 1$-associated PPI network, including ASXL transcriptional regulator 1 (ASXL1), proteasome 26S subunit, non-ATPase 3 (PSMD3), proteasome 26S subunit, non ATPase 11 (PSMD11) and ubiquitin $\mathrm{C}(U B C)$, were statistically significantly associated with the overall survival of patients with melanoma. The expression levels of PRKCA, BUB1,BAP1 and ASXL1 were significantly different between primary melanoma and metastatic melanoma. Based on the present study, 'extracellular exosome' processes, 'amoebiasis' pathways, 'ECM-receptor interaction' pathways and 'focal adhesion' signaling pathways may be important in the formation of metastases from melanoma. The involved genes, including PIK3R3, CENPM, AURKA, LAMA1, PCNA, ADCY1, $B U B 1, N D C 80$ and $P R K C A$, and mutation associated genes, including $B A P 1, A S X L 1, P S M D 3, P S M D 11$ and $U B C$, may serve important roles in metastases of melanoma.

\section{Introduction}

Melanoma is a common malignancy and has one of the poorest prognoses due to its excessive aggression and metastasis (1). In recent years, the incidence of melanoma has increased significantly and the survival rate of patients with melanoma remains poor (2). As metastasis is the most lethal stage of melanoma progression, identifying its underlying molecular mechanisms and determining its molecular biomarkers is important.

Microarray analysis is able to quickly identify all of the genes expressed at any one time-point and is particularly suited to screen for differentially expressed genes (DEGs) (3). Recently, microarray analysis has been widely applied and has produced large amounts of microarray data, which are deposited in public databases. It may be beneficial for further research to integrate and reanalyze this data (4). 
In a previous study, the microarray dataset GSE8401 generated by $\mathrm{Xu}$ et al (5) was used to validate a 'metastasis aggressiveness gene expression signature' derived from human melanoma cells, selected based on their metastatic potential in an immunodeficient mouse metastasis model, and their correlation with the aggressiveness of melanoma metastases in human patients. However, an integral bioinformatics analysis combined with an expression profile analysis has not been yet been conducted, to the best of the authors' knowledge.

In the present study, the DEGs from the GSE8401 dataset were identified, subsequent to data preprocessing with the affy package, followed by Gene Ontology and Kyoto Encyclopedia of Genes and Genomes (KEGG) pathway enrichment analyses. A protein-protein interaction (PPI) network was constructed. Mutated genes were analyzed with 80 mutated cases with melanoma from The Cancer Genome Atlas (TCGA). The overall survival of key candidate DEGs, which were within a filtering of degree $>30$ criteria in the PPI network and involved three or more KEGG signaling pathways, and genes with a high mutation frequency were delineated. The expression analysis of key candidate DEGs, mutant genes and their associated genes were performed on UALCAN. Of the 1,187 DEGs obtained, 505 were upregulated and 682 were downregulated. 'Extracellular exosome' processes, the 'amoebiasis' pathway, the 'ECM-receptor interaction' pathway and the 'focal adhesion' signaling pathway were significantly enriched and identified as important processes or signaling pathways. The overall survival analysis of phosphoinositide-3-kinase regulator subunit 3 (PIK3R3), centromere protein $\mathrm{M}(C E N P M)$, aurora kinase A (AURKA), laminin subunit $\alpha 1$ (LAMA1), proliferating cell nuclear antigen ( $P C N A)$, adenylate cyclase 1 ( $A D C Y 1)$, BUB1 mitotic checkpoint serine/threonine kinase (BUB1), NDC80, kinetochore complex component (NDC80) and protein kinase $\mathrm{C} \alpha(P R K C A)$ in DEGs was statistically significant. Mutation gene analysis identified that BRCA1-associated protein $1(B A P 1)$ had a higher mutation frequency and survival analysis, and its associated genes in the $B A P I$-associated PPI network, including ASXL transcriptional regulator 1 (ASXL1), proteasome 26S subunit, non-ATPase 3 (PSMD3), proteasome 26S subunit, non ATPase 11 (PSMD11) and ubiquitin C $(U B C)$, were statistically significantly associated with the overall survival of patients with melanoma. The expression levels of PRKCA, BUB1, BAPI and ASXL1 were significantly different between primary melanoma and metastatic melanoma. Based on the present study, 'extracellular exosome' processes, 'amoebiasis' pathways, 'ECM-receptor interaction' pathways and 'focal adhesion' signaling pathways may be important in the formation of metastases from melanoma. The involved genes, including PIK3R3, CENPM, AURKA, LAMA1, PCNA, $A D C Y 1, B U B 1, N D C 80$ and $P R K C A$, and mutation associated genes, including $B A P 1, A S X L 1, P S M D 3, P S M D 11$ and $U B C$, may serve important roles in metastases of melanoma.

\section{Materials and methods}

Affymetrix microarray data and clinical information. The gene expression profile dataset GSE8401 was extracted from the Gene Expression Omnibus (GEO) database (https://www.ncbi.nlm.nih.gov/geo/) (6). The GSE8401 dataset was deposited by $\mathrm{Xu}$ et al (5), including 52 biopsy specimens from patients with melanoma metastasis and 31 biopsy specimens from patients with primary melanoma, and was based on the platform of the GPL96 (HG-U133A) Affymetrix Human Genome U133A Array (Affymetrix; Thermo Fisher Scientific, Inc., Waltham, MA, USA). These 83 fresh melanoma biopsies from patients undergoing surgery were collected between 1992 and 2001 as a part of the diagnostic or therapeutic strategy. Immediately following surgery, half of each specimen was fixed in formalin and processed for routine histology, and the other half was immediately snap-frozen and stored in liquid nitrogen until use for RNA extraction. Histopathological diagnosis of each tissue specimen was performed independently by two histopathologists. All patient specimens were collected and used, according to the approval by the institutional ethics committee and written informed consent was obtained, according to the ethical standards in the 1964 Declaration of Helsinki.

Data preprocessing and DEG screening. The downloaded data in the series matrix file was preprocessed using the affy package (version 1.50.0) (7) in R language, including normalization and expression calculation. Annotations to the probes were performed, and probes that were not matched to the gene symbols were excluded. The average expression values were taken if different probes mapped to the same gene. DEGs in patients with melanoma metastasis compared with patients with primary melanoma were analyzed using the limma package (8) in $\mathrm{R}$ language. The cut-off threshold was set as a $\mathrm{P}<0.05$ and $\log _{2}$ (fold change) $>1$ or $<-1$.

GO and pathway enrichment analysis. GO (http://www. geneontology.org/) analysis is commonly used for functional studies of large-scale genomic or transcriptomic data and classifies functions with respect to three criteria: Molecular function (MF), cellular component (CC) and biological process (BP) $(9,10)$. The KEGG (http://www.kegg.jp/) pathway database is widely used for the systematic analysis of gene functions, associating genomic data with higher order functional data (11). The Database for Annotation, Visualization and Integrated Discovery (DAVID; http://david.ncifcrf.gov/) is an integrated biological knowledge base with analytical tools used for the systematic and integrative analysis of large gene lists (12). In the present study, GO terms and KEGG signaling pathway enrichment analyses for DEGs were performed using DAVID. The cutoff threshold was set as $\mathrm{P}<0.05$.

Integration analysis of the PPI network and the KEGG pathway network. Search Tool for the Retrieval of Interacting Gene/Proteins (STRING; http://www.string-db.org/) is an online database that assesses and integrates PPIs (13). In the present study, DEGs were mapped onto the STRING database for PPI analysis, with a combined score of 0.4 as the parameter setting. The PPI network generated with the DEGs was constructed with Cytoscape software (version 3.4.0) $(14,15)$ and the topology scores of the nodes in the PPI network were analyzed using the CentiScape plugin (version 3.4.0; Parameter setting: Without weight) (16). The key candidate 
genes were screened with a filtering of degree $>30$ criteria in the PPI network. Networks of significantly enriched $(\mathrm{P}<0.05)$ KEGG pathways and their associated genes were constructed using the Cytoscape (version 3.4.0) plugin ClueGO (14) and Cluepedia (15). The cross-talk genes were identified with the filtering criteria of association with at least three signaling pathways.

Mutantgenesscreening.ThecBioPortal(http://www.cbioportal. org/) for Cancer Genomics, which provides the visualization, analysis and download of large-scale cancer genomics datasets, was employed for the mutation analysis $(17,18)$. In total, 80 mutated cases were selected from TCGA database (project ID: TCGA_SKCM; https://cancergenome.nih.gov/) to screen for mutated genes (19). The detailed clinical information of the samples is listed in Table I.

Overall survival and expression analysis. Overall survival analyses for key candidate DEGs and mutation associated genes were performed using Gene Expression Profiling Interactive Analysis (http://gepia.cancer-pku.cn/index.html) (20). Briefly, by entering the genes of interest to the website, patients were divided into two groups according to the expression level of gene, and the survival rate was statistically analysed. The hazard ratio (HR) with $95 \%$ confidence intervals and log rank $\mathrm{P}$-value were calculated and are presented. The expression analysis of key candidate DEGs and mutation-associated genes in melanoma was performed using UALCAN (http://ualcan. path.uab.edu/analysis.html) (21).

\section{Results}

Data normalization and analysis of DEGs. The boxplot demonstrated good normalization of the GSE8401 data (Fig. 1A). In total, 22,283 probes were obtained, among which 1,205 probes were differentially expressed. Following annotation, 1,187 DEGs in patients with melanoma metastasis compared with patients with primary melanoma were identified, including 505 upregulated DEGs and 682 downregulated DEGs (Fig. 1B).

GO and signaling pathway enrichment analysis. GO enrichment analysis for the DEGs was performed. The top 10 most significant GO terms of each group are presented in Fig. 2A. For MF, the DEGs were primarily enriched in 'structural molecule activity' processes. For CC, the DEGs were primarily enriched in 'extracellular exosome' processes and 'extracellular space' processes. For BP, the DEGs were primarily enriched in 'epidermis development' processes, 'keratinocyte differentiation' processes and 'cell adhesion' processes.

The top 20 most significant KEGG pathway terms are presented in Fig. 2B. The genes were primarily enriched in 'amoebiasis', 'ECM-receptor interaction' and 'focal adhesion' signaling pathways.

PPI analysis. Using the STRING online database and Cytoscape software, a total of 447 DEGs of the 1,187 DEGs were mapped onto the PPI network complex (Fig. 3), and 740 of 1,187 DEGs did not fall into the PPI network.
Cross-talk genes and key candidate gene screening. The cross-talk genes were identified with the filtering criteria of association with at least three signaling pathways, based on the KEGG pathway analysis (Fig. 4). The key candidate genes were screened, including PIK3R3, CENPM, AURKA, LAMA1, $P C N A, A D C Y 1, B U B 1$, NDC80 kinetochore complex component (NDC80) and PRKCA. Among these genes, PIK3R3, $P R K C A, A D C Y 1$ and $L A M A 1$ were enriched in the 'amoebiasis' signaling pathway. $A D C Y 1$ was additionally involved in 'pancreatic secretion', 'GnRH signaling pathway', 'melanogenesis' and 'pathways in cancer' signaling pathways. The majority of these genes were involved in 'pathways in cancer', 'ECM-receptor interaction' and 'focal adhesion' signaling pathways.

Overall survival analysis of key candidate genes. The survival analysis sourced from TCGA data was performed and the analysis curves demonstrated that the aberrant expressions of the key candidate genes, including $P I K 3 R 3$, CENPM, AURKA, LAMA1, PCNA, ADCY1, BUB1, NDC80 and $P R K C A$, were significantly associated with poor overall survival (Fig. 5).

Mutant genes and their associated gene analysis. The mutation analysis of genes was performed on cBioPortal based on 80 patients with melanoma from TCGA database. The results demonstrated that $\mathrm{G}$ protein subunit $\alpha \mathrm{q}, \mathrm{G}$ protein subunit $\alpha 11, B A P 1$ and slicing factor $3 b$ subunit 1 had the highest mutation frequency (Fig. 6). Only the survival analysis of BAP1 was statistically significant (data not shown).

A BAP1-associated PPI network was constructed (Fig. 7), in which the survival analyses of ASXL1, proteasome $26 \mathrm{~S}$ subunit, non-ATPase 3 (PSMD3), proteasome 26S subunit, non ATPase 11 (PSMD11) and UBC were statistically significant (Fig. 8). Survival analysis was conducted in 40 melanoma cases with metastasis selected from the 80 patients with melanoma in the TCGA database (14 cases with BAP1 mutations and 26 cases without BAP1 mutations) to identify the prognostic value of BAP1 mutations in melanoma cases with metastasis (Fig. 9); information on the cases is provided in Table I.

\section{Discussion}

The majority of patients with melanoma are diagnosed at advanced stages and have poor overall survival (22). However, the molecular mechanisms involved in the metastasis and progression of malignant melanoma remain unclear.

In the present study, the raw gene expression data of GSE8401 was obtained from the GEO. The original previous study identified that membrane proteins served an important role in tumor-microenvironment interactions during metastasis (5). However, further investigation is required regarding the clinical value of these genes. In the present study, a comprehensive bioinformatics analysis based on the GSE8401 dataset and TCGA was performed. The results suggested that high expression of PIK3R3, CENPM, AURKA, LAMA1, PCNA, ADCY1, BUB1, NDC80 and PRKCA in patients with melanoma was associated with poor overall survival. 
Table I. Information of the cases with metastasis.

A, Deceased patients

\begin{tabular}{lccr}
\hline Submitter ID & BAPI mutation & Tumor stage & Days to mortality \\
\hline TCGA-WC-A88A & Yes & IV & 82 \\
TCGA-WC-A884 & Yes & IIIb & 123 \\
TCGA-WC-A883 & Yes & IIIa & 241 \\
TCGA-WC-A87U & Yes & IIIa & 581 \\
TCGA-VD-A8KF & No & IIIb & IV \\
TCGA-VD-A8KD & No & IIIa & 114 \\
TCGA-V4-A9EX & No & IIIa & 730 \\
TCGA-V4-A9EV & No & IIIa & 1,105 \\
TCGA-V4-A9ES & Yes & IIIa & 511 \\
TCGA-V4-A9EQ & Yes & IIIb & 355 \\
TCGA-V4-A9EI & No & IIIa & 389 \\
TCGA-V4-A9E9 & Yes & IIIa & 1,246 \\
TCGA-V4-A9E8 & Yes & IIIa & IV \\
TCGA-V4-A9E7 & No & & 558 \\
TCGA-RZ-AB0B & No & & 349 \\
\hline
\end{tabular}

$\mathrm{B}$, Alive patients

\begin{tabular}{|c|c|c|c|}
\hline Submitter ID & $B A P 1$ mutation & Tumor stage & Days to last follow-up \\
\hline TCGA-YZ-A985 & Yes & IIIa & 1,184 \\
\hline TCGA-YZ-A982 & Yes & $\mathrm{IIIb}$ & 895 \\
\hline TCGA-YZ-A980 & Yes & IIIa & 862 \\
\hline TCGA-WC-A880 & Yes & IIIa & 1,258 \\
\hline TCGA-VD-A8KK & Yes & IIIa & 624 \\
\hline TCGA-VD-A8KH & No & $\mathrm{IIIb}$ & 1,158 \\
\hline TCGA-VD-A8K7 & No & IIIa & 1,459 \\
\hline TCGA-V4-A9F8 & No & IIIa & 797 \\
\hline TCGA-V4-A9F5 & No & IV & 603 \\
\hline TCGA-V4-A9F4 & No & IIIa & 634 \\
\hline TCGA-V4-A9F3 & No & IIIa & 1543 \\
\hline TCGA-V4-A9F2 & No & IIIa & 934 \\
\hline TCGA-V4-A9F0 & No & IIIb & 1,678 \\
\hline TCGA-V4-A9EY & No & IIIa & 837 \\
\hline TCGA-V4-A9EW & No & IIIa & 1,211 \\
\hline TCGA-V4-A9EU & No & IIIc & 709 \\
\hline TCGA-V4-A9ET & No & $\mathrm{IIIb}$ & 1,362 \\
\hline TCGA-V4-A9EO & Yes & IIIa & 973 \\
\hline TCGA-V4-A9EM & No & IIIa & 966 \\
\hline TCGA-V4-A9EL & No & IIIa & 1,082 \\
\hline TCGA-V4-A9EH & No & $\mathrm{IIIb}$ & 670 \\
\hline TCGA-V4-A9EF & No & $\mathrm{IIIb}$ & 1,165 \\
\hline TCGA-V4-A9ED & No & IIIa & 1,078 \\
\hline TCGA-V4-A9EA & No & IIIa & 1,348 \\
\hline TCGA-V4-A9E5 & No & $\mathrm{IIIb}$ & 1,799 \\
\hline
\end{tabular}

TCGA, The Cancer Genome Atlas; BAP1, BRCA1-associated protein 1.

PRKCA serves a critical role in the growth of human melanoma cells and a low expression level of PRKCA promotes cellular proliferation (23). In models of melanoma, activation of PRKCA was associated with increased tumor cell prolifera- 

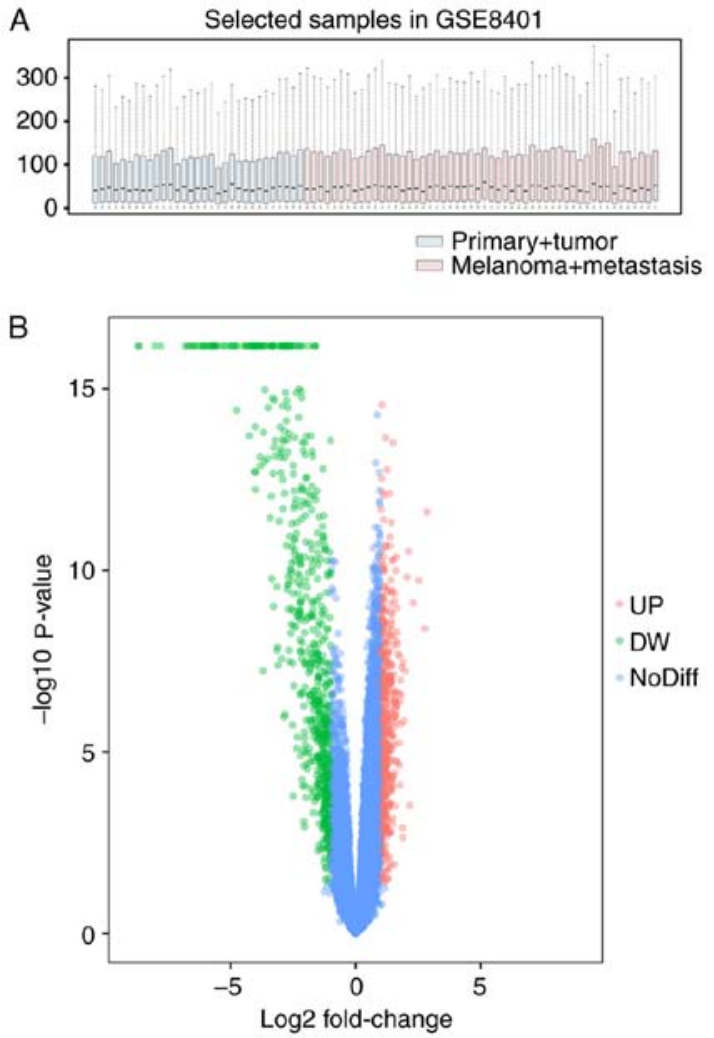

Figure 1. Data normalization and identification of differentially expressed genes in GSE8401. (A) Boxplot for normalized gene expression data and (B) volcano plot of significantly differentially expressed genes. UP, upregulated; DW, downregulated; NoDiff, no difference.

tion and invasiveness (24). Small interfering RNA-mediated knockdown of $\underline{P R K C A}$ resulted in a significant suppression of invasion (25). $A D C Y 1$, a member of the adenylate cyclase gene family, encodes adenylate cyclase. $A D C Y 1$ demonstrated a downregulated trend in rectal adenocarcinoma metastasis (26). LAMA 1 expression was increased in esophageal squamous cell carcinoma tissues, and its overexpression rescued the multiplication suppression and cell apoptosis increases induced by microRNA-202 (27).

In the present study, PRKCA, ADCYI and LAMAI were enriched in the 'amoebiasis' signaling pathway. Colonic amoebiasis has been commonly demonstrated to mimic colon carcinoma (28-30). A rare case of colonic amoebiasis was identified to coexist with signet-ring cell carcinoma (31). All of these results support the hypothesis that PRKCA may contribute to the metastases of melanoma.

A previous study demonstrated that ADCY1 had a downregulated trend in rectal adenocarcinoma metastasis and was significantly enriched in the 'pancreatic secretion' pathway (26). In the present study, ADCY1 was identified to be involved in several melanoma-related pathways such as 'pathways in cancer' and 'melanogenesis'. Previous study has shown that melanogenesis plays a key role in regulation of cellular metabolism of melanoma cell (32).

The diagnostic gene $B U B 1$, a discriminator between melanoma, benign nevi and lymph nodes, contributes to the detection of melanoma micrometastasis in sentinel lymph nodes (33). Utilizing gene microarray analysis and real-time quantitative polymerase chain reactions, the expression level of $B U B 1$ has been identified to be high in metastatic melanoma (34). BUB1 is downstream of sirtuin 1 (SIRT1), which is upregulated in human melanoma, and its inhibition causes an anti-proliferative effect in melanoma cells (35). The expression of $B U B 1$ is suppressed by the inhibition of SIRT1 (35). Therefore, high expression of $B U B 1$ promotes melanoma metastasis and results in a poor prognosis.

$A U R K A$ is known to induce the formation of the mitotic spindle and to be involved in cell multiplication and migration (22). AURKA was upregulated in malignant melanoma cells and tissues, and $A U R K A$ overexpression promoted human melanoma cell growth (22). Activation of $A U R K A$ is driven by the forkhead box protein M1 and the mitogen-activated protein kinase/extracellular-regulated kinase signaling pathway, and is associated with a poor prognosis in patients with melanoma (36). A comprehensive gene expression analysis of melanoma metastasis suggested that $B U B 1$ and $A U R K A$ may serve crucial roles in the development and progression of melanoma (37).

NDC80 is a novel oncogene that is involved in the occurrence of various tumors. It is highly expressed in a variety of cancer types, including pancreatic cancer, hepatocellular carcinoma, gastric cancer and colorectal cancer (38-41). Overexpression of NDC80 in cancer cells and tissues promoted multiplication and migration, and was correlated with poor outcomes in those patients with pancreatic cancer (38). In patients with colorectal cancer, NDC80 overexpression was significantly associated with advanced tumor stage and a poor prognosis (41). NDC80 is a downstream gene of polo-like kinase 1 , which is a well-documented oncogene that serves a key role in cell reproduction and invasion (42). In bladder cancer, NDC80 was associated with the regulation of cell reproduction, migration and invasion, and was positively associated with metastasis (43). All of these results suggested that NDC80 serves an important role in the metastases of melanoma.

PIK3R3, which activates the protein kinase B/serine/threonine-protein kinase mTOR signaling pathway, serves a crucial role in the survival, proliferation and motility of various tumors $(44,45)$. PIK3R3 is involved in the regulation of the actin cytoskeleton and regulates triple-negative breast cancer cell migration (46). Its expression is high in human hepatocellular carcinoma tissues and glioma tissues $(45,47,48)$. PIK3R3 overexpression augments tumor migration and aggressiveness, and promotes the metastasis of colorectal cancer (49). The basal expression level of PIK3R3 was upregulated in highly metastatic human non-small-cell lung cancer cells (44).

PCNA is a proliferation marker and regulates cell growth (50). In esophageal tumor tissues, upregulation of MAGE family member D1 promoted cell growth by interacting with PCNA (51). CENPM and $U B C$ were additionally identified as key candidate genes in the present study. However, $C E N P M$ and $U B C$ have not been previously identified to be involved in cancer. Their function and roles require further experimental verification.

In addition, it was identified that the somatic mutation frequency of $B A P l$ was high in patients with melanoma and patients with a low expression level of $B A P l$ had poor overall survival. Conversely, high expression of ASXL1, PSMD3, 


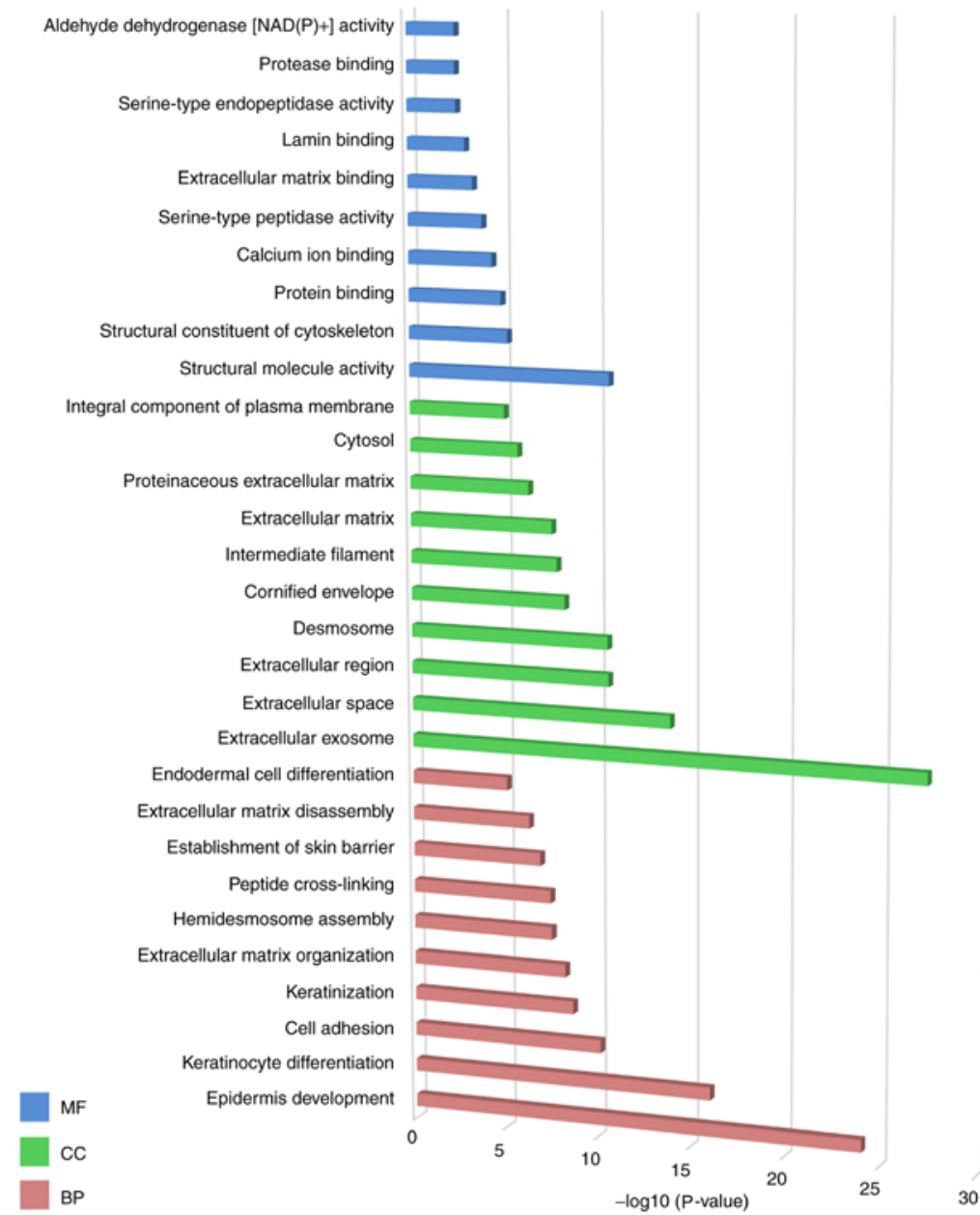

B

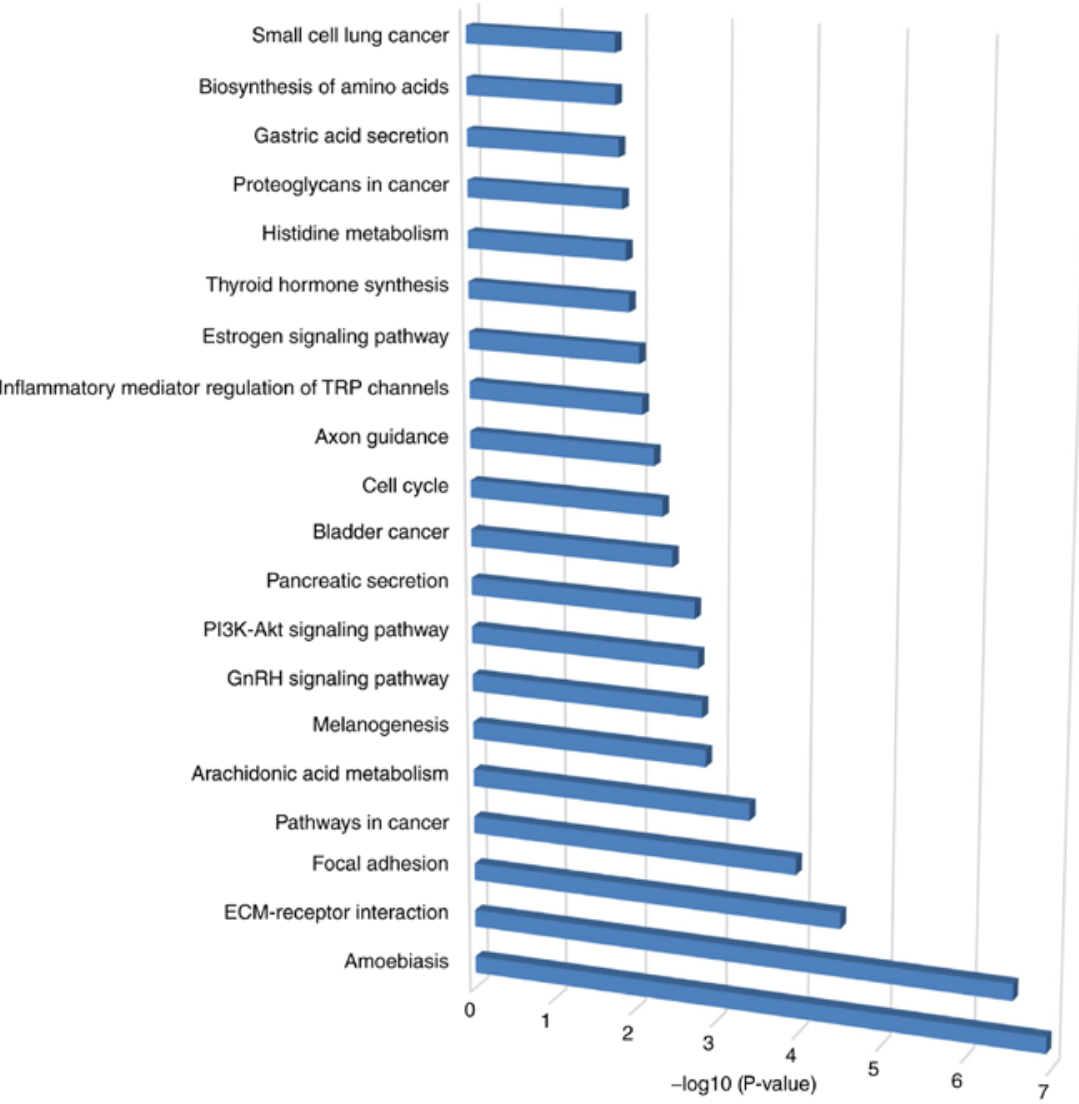

Figure 2. Gene Ontology and signaling pathway enrichment analysis. (A) Gene Ontology and (B) signaling pathway enrichment analysis of differentially expressed genes. MF, molecular function; $\mathrm{CC}$, cellular component; $\mathrm{BP}$, biological process. 


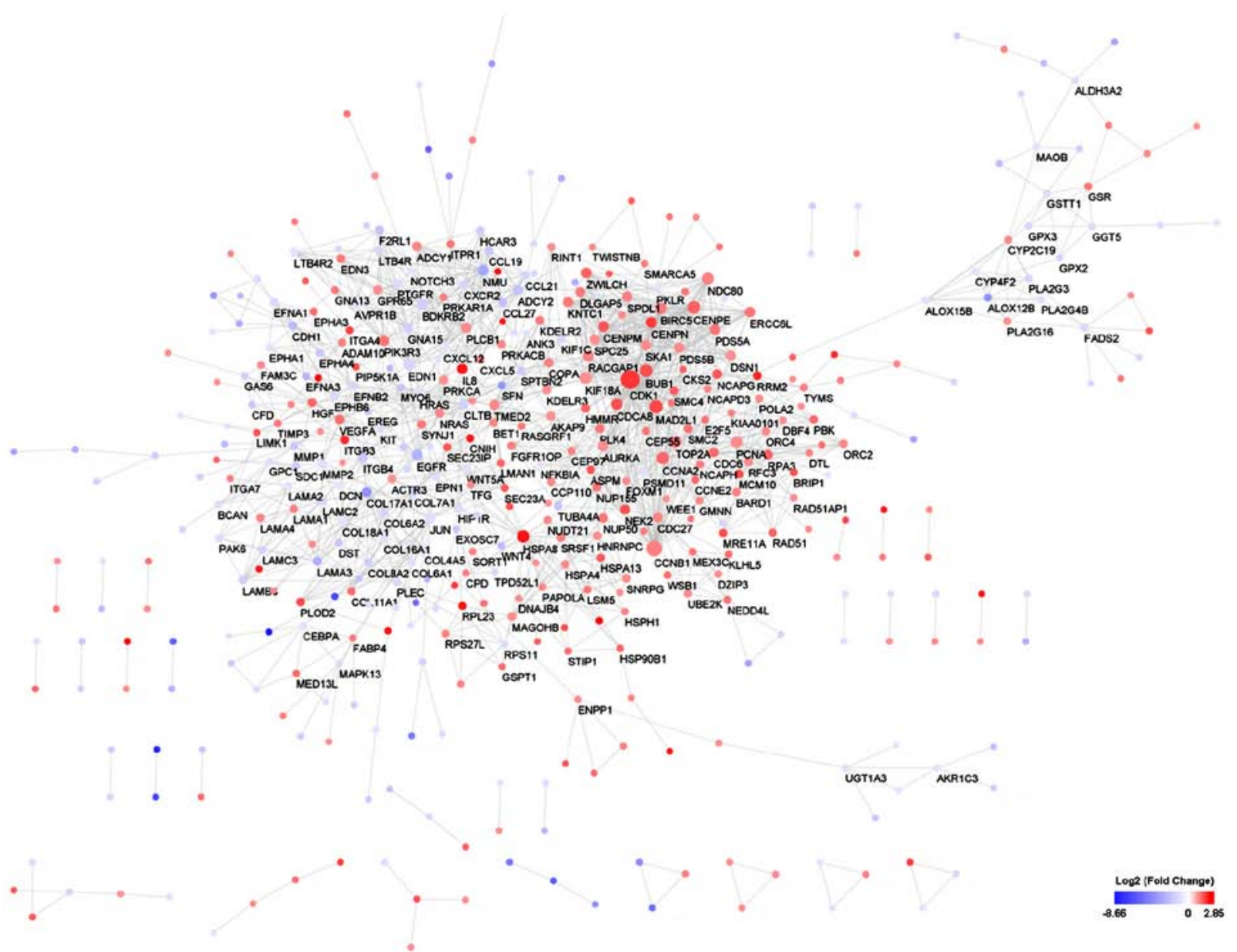

Figure 3. Differentially expressed gene protein-protein interaction network. Red nodes represent upregulated genes and blue nodes represent downregulated genes. The more the degree of the node, the bigger the nodes.

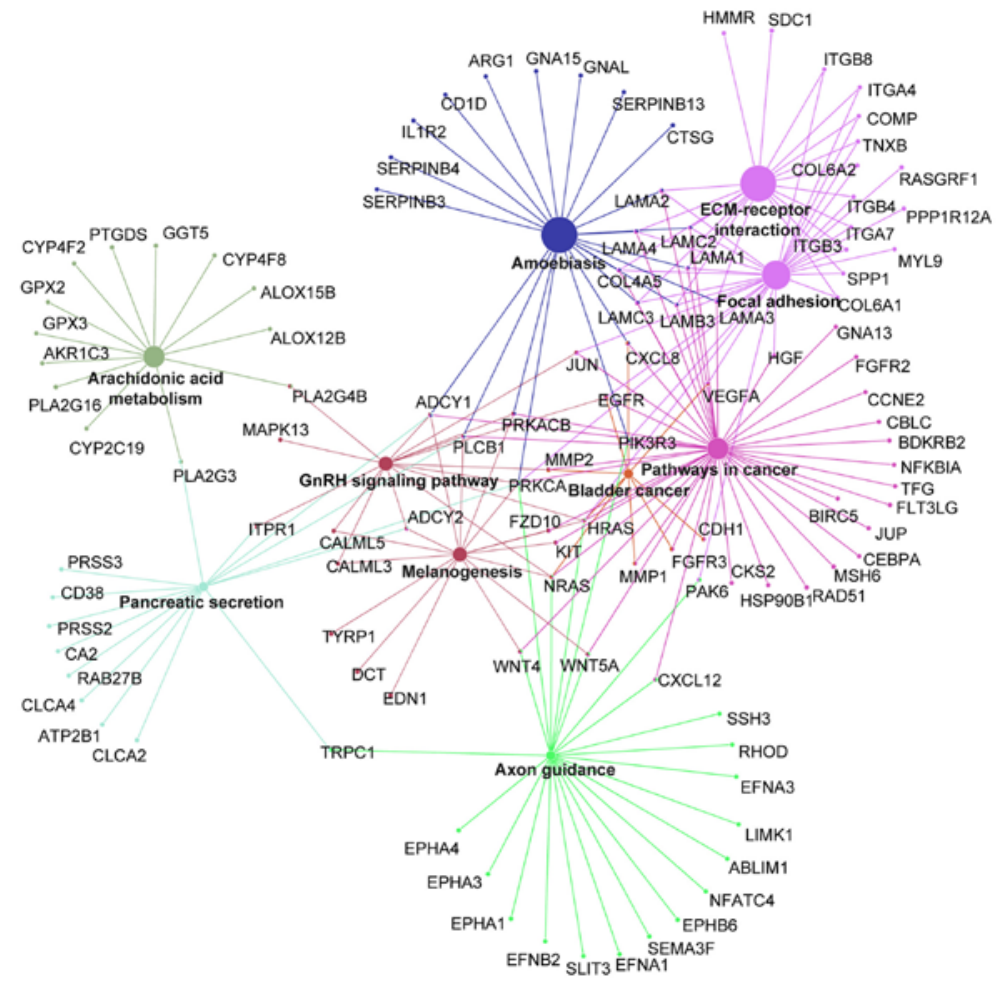

Figure 4. Differentially expressed gene Kyoto Encyclopedia of Genes and Genomes network analysis. The larger size of the nodes represent larger enrichment scores and different colors represent the different enrichment modules. 

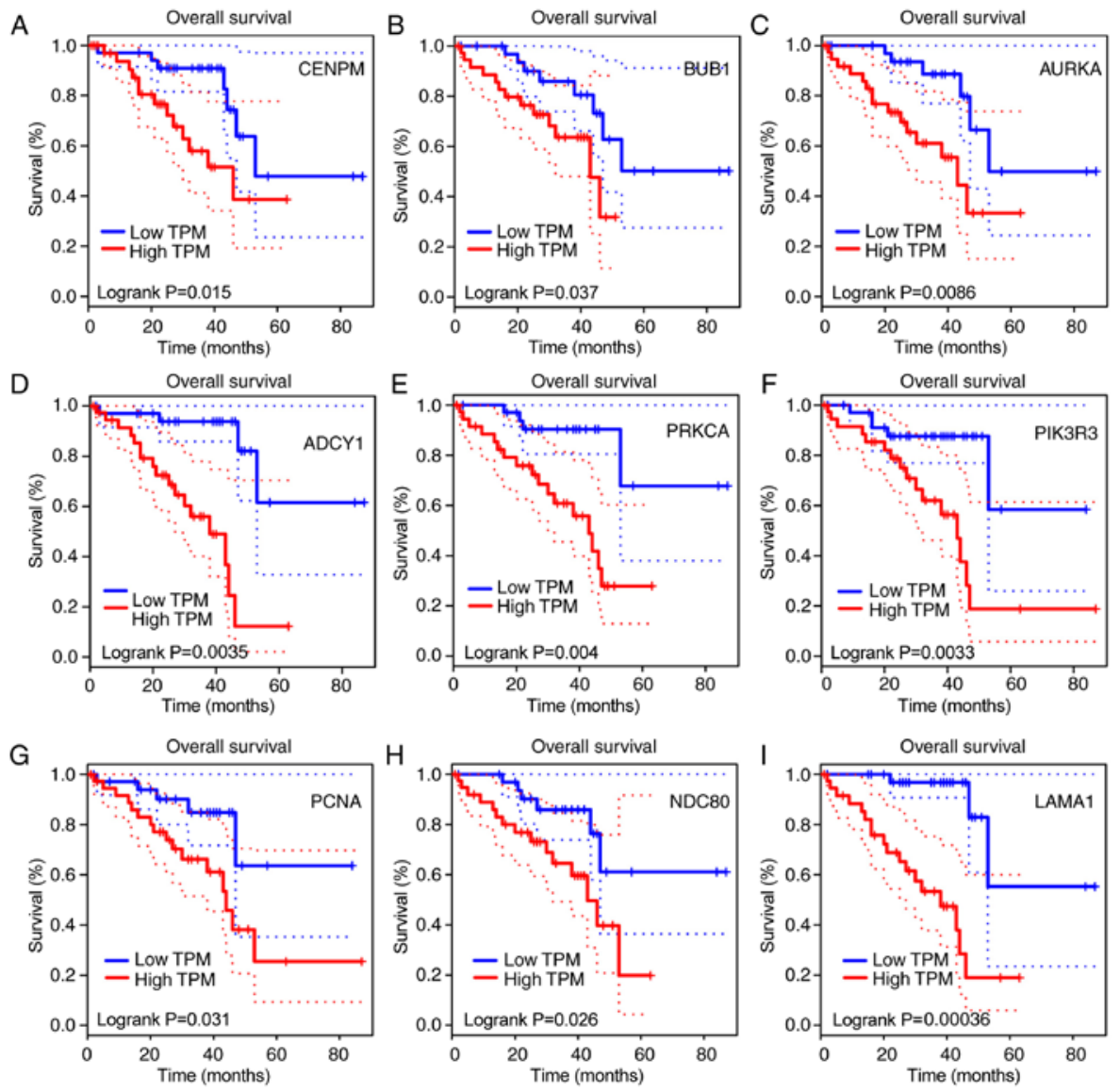

Figure 5. Overall survival curves of key candidate genes in the protein-protein network. Overall survival curves of (A) CENPM, (B) BUB1,(C) $A U R K A$, (D) $A D C Y 1$, (E) PRKCA, (F) PIK3R3, (G) PCNA, (H) NDC80 and (I) LAMA1. CENPM, centromere protein M; BUB1, BUB1 mitotic checkpoint serine/threonine kinase; $A U R K A$, aurora kinase A; $A D C Y 1$, adenylate cyclase 1; PRKCA, protein kinase $\mathrm{C} \alpha ; P I K 3 R 3$, phosphoinositide-3-kinase regulator subunit 3; PCNA, proliferating nuclear antigen; NDC80, NDC80 kinetochore complex component; LAMA1, laminin subunit $\alpha$ 1; TPM, transcripts per million.

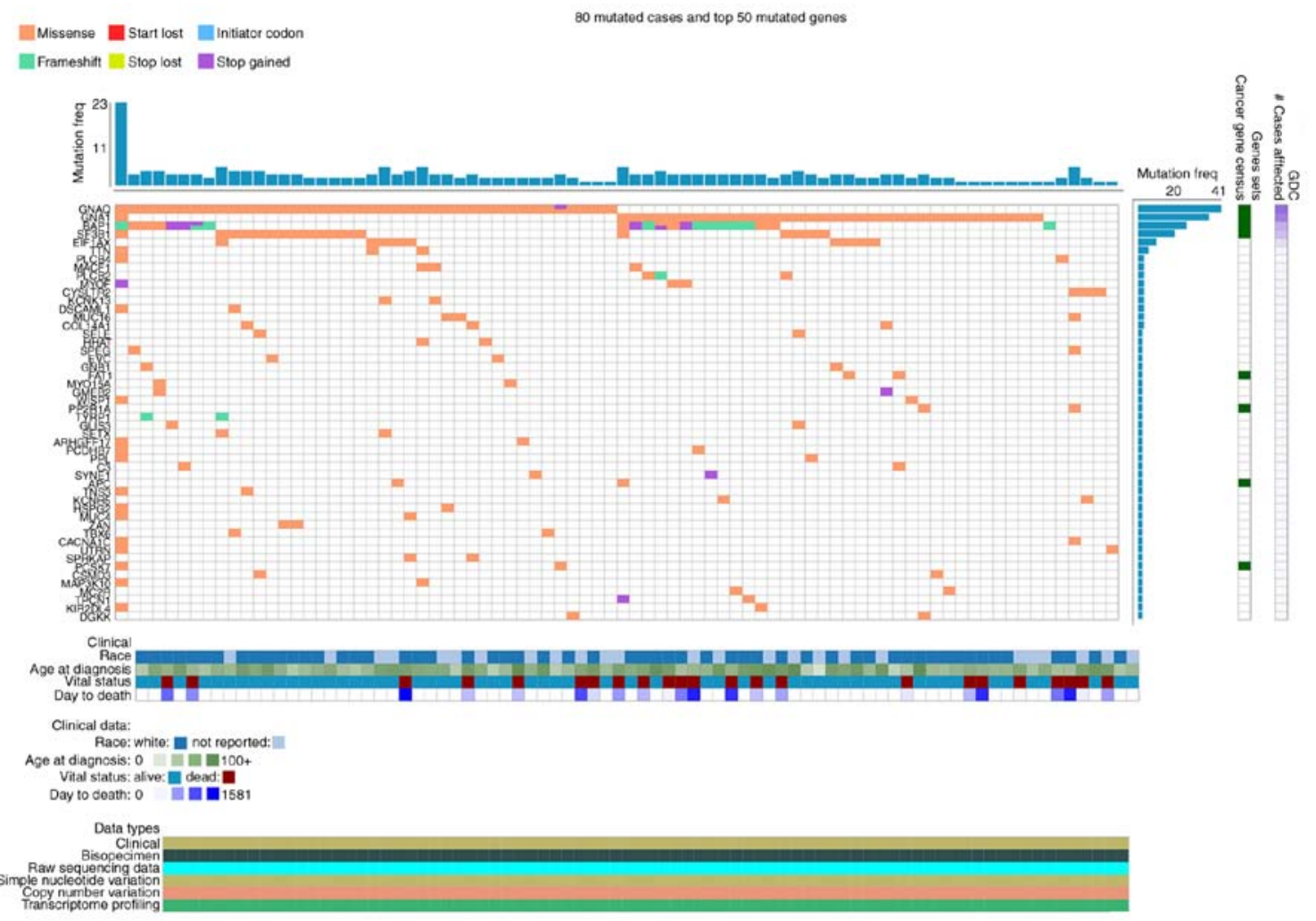

Figure 6. Top 50 mutated genes in 80 mutated cases with melanoma. 


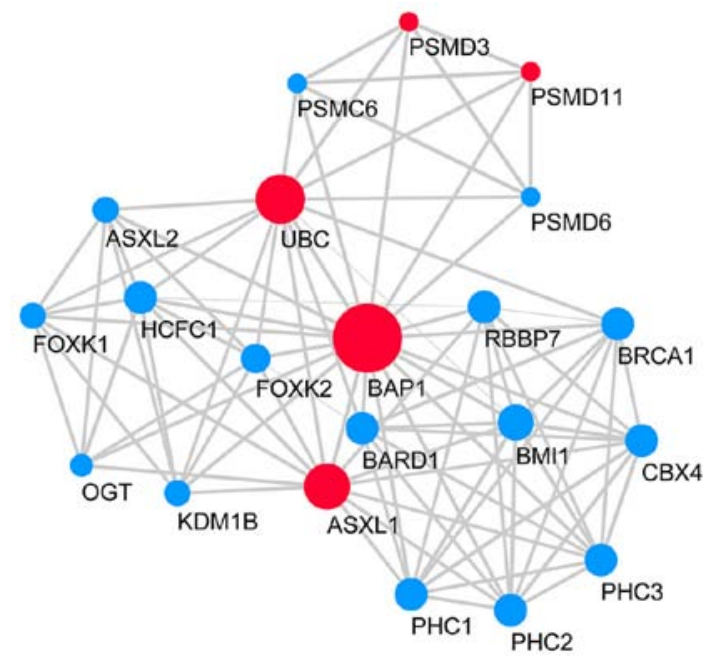

Figure 7. BAPl-associated gene PPI network. Red nodes represent upregulated genes and blue nodes represent downregulated genes. The larger size of the nodes represent more degrees in the global PPI network and lines indicate interactions. BAP1, BRCA1 associated protein 1; PPI, protein-protein interaction.

$P S M D 11$ and $U B C$, which are $B A P 1$-associated genes, was associated with poor overall survival.

$B A P 1$ is a novel identified tumor suppressor gene and somatic mutations of $B A P 1$ occur in a variety of malignancies (52). BAPl serves a key role in stabilizing epigenetic regulators, including $\mathrm{O}$-linked $\mathrm{N}$-acetylglucosamine $(G l c N A c)$ transferase, host cell factor $\mathrm{C} 1$ and $A S X L 1$, and thus an ASXL/BAP1 complex may inhibit chronic myelomonocytic leukemia, in which $A S X L 1$ is frequently mutated (53). The frequency of $B A P 1$ mutations may be undervalued, and a number of previously unidentified $B A P I$ and spliceosome mutations were identified in uveal melanoma (54). Inactivating mutations in $B A P l$ were associated with a high metastatic risk of uveal melanoma (55). In the clinical setting, anti-BAP-1 has been used to detect the presence of BAP-1 mutation (56). Histologically, the melanocytic lesions are located predominantly in the dermis and are composed of epithelioid melanocytes with abundant cytoplasm and distinct nucleoli. Similar lesions develop in the context of somatically acquired BAP-1 loss (57). Immunohistochemistry analysis with anti-BAP-1 demonstrated loss of nuclear staining in melanocytic lesions with BAP-1 mutations $(56,58)$. In the present study, patients with low expression levels of $B A P l$ had poor overall survival, and the expression of $B A P 1$ was lower in metastatic melanoma compared with primary melanoma. Moreover, mutation of $B A P 1$ was associated with poor prognosis in 40 melanoma cases with metastasis. These results suggested that mutations of $B A P l$ may serve a key role in the metastases of melanoma.

$A S X L 1$ is involved in polycomb repressive complex 2-dependent transcriptional repression and nuclear hormone receptor- and BAP1-dependent transcriptional regulation. Somatic mutations of $A S X L$ family members occur in human cancer (59). ASXL1 overexpression was identified in cervical cancer (60) and truncation mutations in $A S X L 1$ were identified in various diseases, including chronic lymphocytic leukemia, malignant myeloid diseases, head and neck squamous cell carcinoma, colorectal cancer, liver cancer, prostate cancer and breast cancer $(58,59)$. Furthermore, truncation mutations in $A S X L 1$ resulted in a poor prognosis of myeloid malignancies $(61,62)$. This is inconsistent with the present result that ASXL1 was highly expressed in metastatic melanoma and associated with poor overall survival in patients with melanoma. ASXL1 may have different functions in the metastases of melanoma. This requires further validation.

Silencing of PSMD3 together with human epidermal growth factor receptor 2 resulted in an additive suppression of cell viability, multiplication and protein kinase $B$ signaling pathway activity (63). PSMD3 is downregulated in the long-term survivor subtype of glioblastoma multiforme, suggesting that a low expression level of PSMD3 results in longer survival (64). PSMD11 was overexpressed in breast cancer tissue, and this may be associated with specific biological processes and pathological types of breast cancer (65). PSMD11 is a short-lived protein, and knockdown of PSMD11 via RNA interference partially resulted in the occurrence of acute apoptosis in pancreatic cancer cells (66). It was identified that high expression of PSMD3 and PSMD11 was associated with poor overall survival in patients with melanoma. Therefore, PSMD3 and PSMD11 may be associated with the occurrence of melanoma metastasis.

Among all of the genes discussed, the key candidate genes and the mutated genes that were identified may serve crucial roles in the metastasis of melanoma. These prognostic markers require further study, and the accuracy of diagnosis may be improved based on multiple gene detection. There are specific limitations to the present bioinformatics analysis. Only a single open GEO dataset was included in the present study, considering that there was no suitable dataset for merge analysis and verification. No functional validation was conducted to support the present findings, although a number of the present observations were supported by previous studies. Biological validation is required in future studies. The survival analysis was conducted based on a TCGA dataset with a limited number of cases. The function and role of the candidate genes in the metastases of melanoma requires further validation. Future studies may aim to validate the gene expression in clinical samples. Additionally, the diagnostic value of $B A P 1$ and its associated genes require validation in clinical samples. Further studies are required to investigate the molecular mechanism of the hub genes, selected in the present study, in melanoma metastasis.

In conclusion, 1,187 DEGs were identified in metastatic melanoma compared with primary melanoma. A total of 447 DEGs were mapped onto a PPI network. Based on the PPI network analysis, gene function enrichment analysis, KEGG network analysis and survival analysis, PIK3R3, CENPM, AURKA, LAMA1, PCNA, ADCY1, BUB1, NDC80 and $P R K C A$, which were associated with poor overall survival, may serve key roles in the metastasis and invasion processes of melanoma. Additionally, BAPl and its associated genes may contribute to the metastasis and invasion processes of melanoma. The results of the present study may provide valuable prognostic markers and therapeutic targets for melanoma. Further studies are required to validate the 

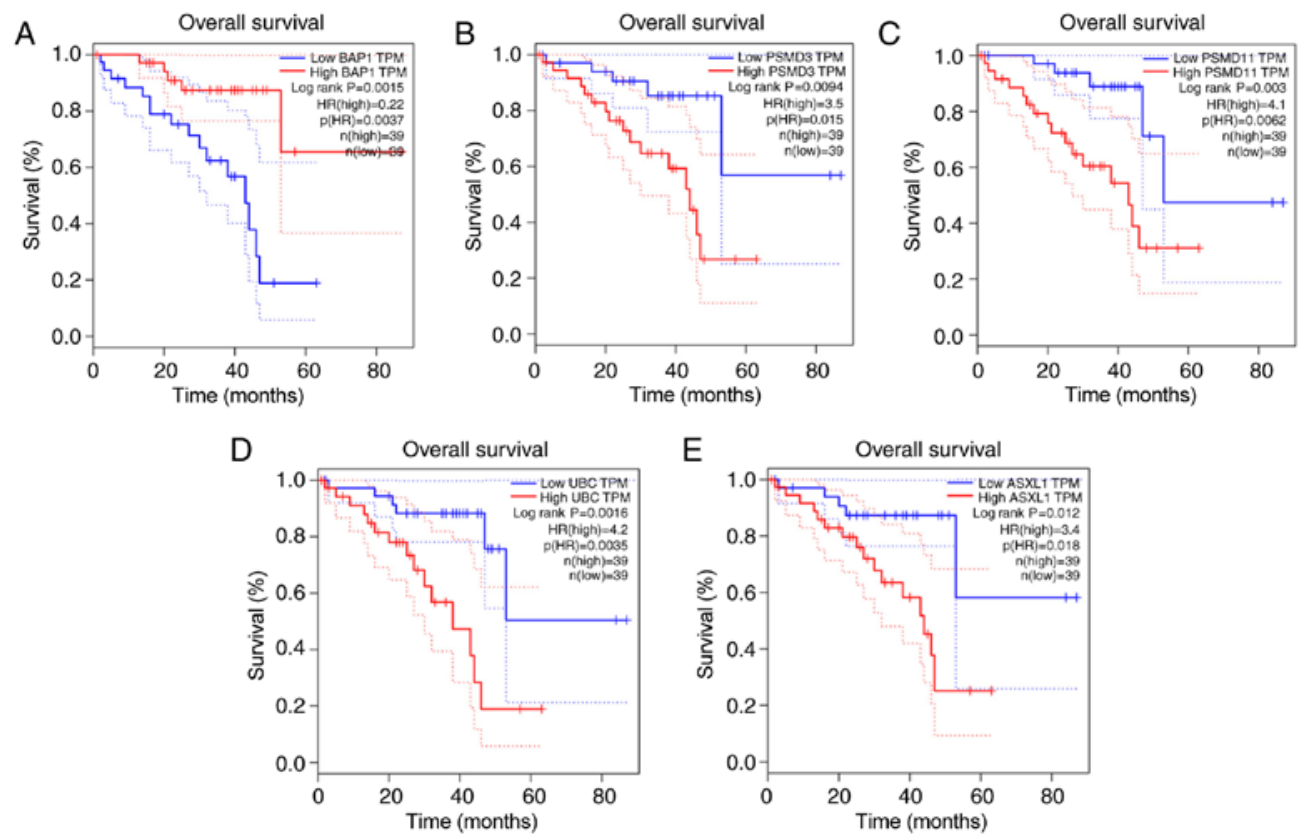

Figure 8. Overall survival curves of BAP1 and its associated genes. Overall survival curves of (A) BAP1, (B) PSMD3, (C) PSMD11, (D) UBC and (E) ASXL1. $B A P 1$, BRCA1 associated protein 1; PSMD3, proteasome 26S subunit, non-ATPase 3; PSMD11, proteasome 26S subunit, non ATPase 11; UBC, ubiquitin C; ASXL1, ASXL transcriptional regulator 1; TPM, transcripts per million; HR, hazard ratio.

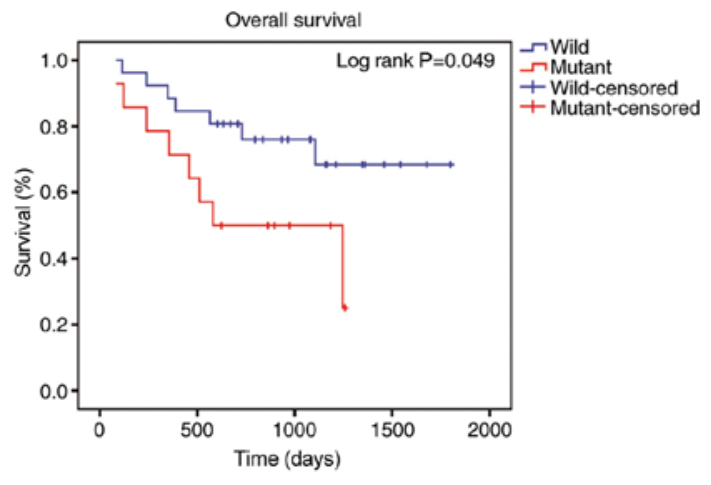

Figure 9. Overall survival curves of associated protein 1 in tumor cases with metastasis.

function and role of these DEGs in the metastasis and aggressiveness of melanoma.

\section{Acknowledgements}

Not applicable.

\section{Funding}

The present study was supported in part by Shanghai Municipal Health Bureau General Project (grant no. 20134381 to Jia Chen) and Science and Technology Commission of Shanghai Municipal Health Bureau Youth Project (grant no. 20114Y057 to Yongxian Lai).

\section{Availability of data and materials}

The datasets used and/or analyzed during the current study are available from the corresponding author on reasonable request.

\section{Authors' contributions}

JC, YLa and YLi conceived and designed the study; JC, FW and YS performed data analysis; FW designed the figures. YS and YLa reviewed the literature. JC and YLi wrote the paper. DY and MX participated in the study design and revised the paper critically for important intellectual content.

\section{Ethics approval and consent to participate}

All patient specimens were collected and used according to the approval by the institutional Ethics Committee, and written informed consent was obtained, according to the ethical standards in the 1964 Declaration of Helsinki.

\section{Patient consent for publication}

Not applicable.

\section{Competing interests}

The authors declare no conflict of interest.

\section{References}

1. Palmieri G, Capone M, Ascierto ML, Gentilcore G, Stroncek DF, Casula M, Sini MC, Palla M, Mozzillo N and Ascierto PA: Main roads to melanoma. J Transl Med 7: 86, 2009.

2. Pessina F, Navarria P, Tomatis S, Cozzi L, Franzese C, Di Guardo L, Ascolese AM, Reggiori G, Franceschini D, Del Vecchio M, et al: Outcome evaluation of patients with limited brain metastasis from malignant melanoma, treated with surgery, radiation therapy, and targeted therapy. World Neurosurg 105: 184-190, 2017.

3. Vogelstein B, Papadopoulos N, Velculescu VE, Zhou S, Diaz LA Jr and Kinzler KW: Cancer genome landscapes. Science 339: 1546-1558, 2013. 
4. Guo Y, Bao Y, Ma M and Yang W: Identification of key candidate genes and pathways in colorectal cancer by integrated bioinformatical analysis. Int J Mol Sci 18: E722, 2017.

5. Xu L, Shen SS, Hoshida Y, Subramanian A, Ross K, Brunet JP, Wagner SN, Ramaswamy S, Mesirov JP and Hynes RO: Gene expression changes in an animal melanoma model correlate with aggressiveness of human melanoma metastases. Mol Cancer Res 6: 760-769, 2008.

6. Edgar R, Domrachev M and Lash AE: Gene Expression Omnibus: NCBI gene expression and hybridization array data repository. Nucleic Acids Res 30: 207-210, 2002.

7. Gautier L, Cope L, Bolstad BM and Irizarry RA: affy-analysis of Affymetrix GeneChip data at the probe level. Bioinformatics 20: 307-315, 2004

8. Smyth GK: Linear models and empirical bayes methods for assessing differential expression in microarray experiments. Stat Appl Genet Mol Biol: https://doi.org/10.2202/1544-6115.1027.

9. Hulsegge I, Kommadath A and Smits MA: Globaltest and GOEAST: Two different approaches for Gene Ontology analysis. BMC Proc 3 (Suppl 4): S10, 2009.

10. Ashburner M, Ball CA, Blake JA, Botstein D, Butler H, Cherry JM, Davis AP, Dolinski K, Dwight SS, Eppig JT, et al; The Gene Ontology Consortium: Gene ontology: Tool for the unification of biology. Nat Genet 25: 25-29, 2000.

11. Kanehisa M and Goto S: KEGG: Kyoto encyclopedia of genes and genomes. Nucleic Acids Res 28: 27-30, 2000.

12. Huang W, Sherman BT and Lempicki RA: Systematic and integrative analysis of large gene lists using DAVID bioinformatics resources. Nat Protoc 4: 44-57, 2009.

13. Szklarczyk D, Franceschini A, Wyder S, Forslund K, Heller D, Huerta-Cepas J, Simonovic M, Roth A, Santos A, Tsafou KP, et al: STRING v10: Protein-protein interaction networks, integrated over the tree of life. Nucleic Acids Res 43 (D1): D447-D452, 2015.

14. Bindea G, Mlecnik B, Hackl H, Charoentong P, Tosolini M, Kirilovsky A, Fridman WH, Pagès F, Trajanoski $Z$ and Galon J: ClueGO: A Cytoscape plug-in to decipher functionally grouped gene ontology and pathway annotation networks. Bioinformatics 25: 1091-1093, 2009.

15. Bindea G, Galon J and Mlecnik B: CluePedia Cytoscape plugin: Pathway insights using integrated experimental and in silico data. Bioinformatics 29: 661-663, 2013.

16. Scardoni G, Petterlini M and Laudanna C: Analyzing biological network parameters with CentiScaPe. Bioinformatics 25 2857-2859, 2009

17. Gao J, Aksoy BA, Dogrusoz U, Dresdner G, Gross B, Sumer SO, Sun Y, Jacobsen A, Sinha R, Larsson E, et al: Integrative analysis of complex cancer genomics and clinical profiles using the cBioPortal. Sci Signal 6: pl1, 2013.

18. Cerami E, Gao J, Dogrusoz U, Gross BE, Sumer SO, Aksoy BA, Jacobsen A, Byrne CJ, Heuer ML, Larsson E, et al: The cBio cancer genomics portal: An open platform for exploring multidimensional cancer genomics data. Cancer Discov 2: 401-404, 2012.

19. Guan J, Gupta R and Filipp FV: Cancer systems biology of TCGA SKCM: Efficient detection of genomic drivers in melanoma. Sci Rep 5: 7857, 2015.

20. Tang Z, Li C, Kang B, Gao G, Li C and Zhang Z: GEPIA: A web server for cancer and normal gene expression profiling and interactive analyses. Nucleic Acids Res 45 (W1): W98-W102, 2017.

21. Chandrashekar DS, Bashel B, Balasubramanya SAH, Creighton CJ, Ponce-Rodriguez I, Chakravarthi BVSK and Varambally S: UALCAN: A Portal for Facilitating Tumor Subgroup Gene Expression and Survival Analyses. Neoplasia 19: 649-658, 2017.

22. Chang X, Zhang H, Lian S and Zhu W: miR-137 suppresses tumor growth of malignant melanoma by targeting aurora kinase $\mathrm{A}$. Biochem Biophys Res Commun 475: 251-256, 2016.

23. Krasagakis K, Lindschau C, Fimmel S, Eberle J, Quass P, Haller $\mathrm{H}$ and Orfanos CE: Proliferation of human melanoma cells is under tight control of protein kinase $\mathrm{C}$ alpha. J Cell Physiol 199: 381-387, 2004

24. Lahn MM and Sundell KL: The role of protein kinase C-alpha (PKC-alpha) in melanoma. Melanoma Res 14: 85-89, 2004.

25. Kabbarah O, Nogueira C, Feng B, Nazarian RM, Bosenberg M, Wu M, Scott KL, Kwong LN, Xiao Y, Cordon-Cardo C, et al: Integrative genome comparison of primary and metastatic melanomas. PLoS One 5: e10770, 2010.
26. Hua Y, Ma X, Liu X, Yuan X, Qin H and Zhang X: Identification of the potential biomarkers for the metastasis of rectal adenocarcinoma. APMIS 125: 93-100, 2017.

27. Meng X, Chen X, Lu P, Ma W, Yue D, Song L and Fan Q: MicroRNA-202 inhibits tumor progression by targeting LAMA1 in esophageal squamous cell carcinoma. Biochem Biophys Res Commun 473: 821-827, 2016.

28. Fernandes H, D'Souza CR, Swethadri GK and Naik CN: Ameboma of the colon with amebic liver abscess mimicking metastatic colon cancer. Indian J Pathol Microbiol 52: 228-230, 2009.

29. Ayari H, Rebii S, Ghariani W, Daghfous A, Hasni R, Rehaiem R, Rezgui-Marhoul L and Zoghlami A: Colonic amoebiasis simulating a cecal tumor: Case report. Med Sante Trop 23: 274-275, 2013 (In French).

30. Moorchung N, Singh V, Srinivas V, Jaiswal SS and Singh G: Caecal amebic colitis mimicking obstructing right sided colonic carcinoma with liver metastases: A rare case. J Cancer Res Ther 10: 440-442, 2014.

31. Grosse A: Diagnosis of colonic amebiasis and coexisting signet-ring cell carcinoma in intestinal biopsy. World J Gastroenterol 22: 8234-8241, 2016.

32. Slominski A, Kim TK, Brożyna AA, Janjetovic Z, Brooks DL, Schwab LP, Skobowiat C, Jóźwicki W and Seagroves TN: The role of melanogenesis in regulation of melanoma behavior: Melanogenesis leads to stimulation of HIF-1 $\alpha$ expression and HIF-dependent attendant pathways. Arch Biochem Biophys 563: 79-93, 2014

33. Lewis TB, Robison JE, Bastien R, Milash B, Boucher K, Samlowski WE, Leachman SA, Dirk Noyes R, Wittwer CT, Perreard L and Bernard PS: Molecular classification of melanoma using real-time quantitative reverse transcriptase-polymerase chain reaction. Cancer 104: 1678-1686, 2005.

34. Riker AI, Enkemann SA, Fodstad O, Liu S, Ren S, Morris C, Xi Y, Howell P, Metge B, Samant RS, et al: The gene expression profiles of primary and metastatic melanoma yields a transition point of tumor progression and metastasis. BMC Med Genomics 1: 13, 2008

35. Singh CK, George J, Nihal M, Sabat G, Kumar R and Ahmad N: Novel downstream molecular targets of SIRT1 in melanoma: A quantitative proteomics approach. Oncotarget 5: 1987-1999, 2014.

36. Puig-Butille JA, Vinyals A, Ferreres JR, Aguilera P, Cabré E, Tell-Martí G, Marcoval J, Mateo F, Palomero L, Badenas C, et al: AURKA Overexpression Is Driven by FOXM1 and MAPK/ERK Activation in Melanoma Cells Harboring BRAF or NRAS Mutations: Impact on Melanoma Prognosis and Therapy. J Invest Dermatol 137: 1297-1310, 2017.

37. Quan L, Shi J, Tian Y, Zhang Q, Zhang Y, Zhang Y, Hui Q and Tao K: Identification of potential therapeutic targets for melanoma using gene expression analysis. Neoplasma 62: 733-739, 2015.

38. Meng QC, Wang HC, Song ZL, Shan ZZ, Yuan Z, Zheng Q and Huang XY: Overexpression of NDC80 is correlated with prognosis of pancreatic cancer and regulates cell proliferation. Am J Cancer Res 5: 1730-1740, 2015.

39. Qu Y, Li J, Cai Q and Liu B: Hec1/Ndc80 is overexpressed in human gastric cancer and regulates cell growth. J Gastroenterol 49: 408-418, 2014

40. Chen ZH, Wang WT, Huang W, Fang K, Sun YM, Liu SR, Luo XQ and Chen YQ: The IncRNA HOTAIRM1 regulates the degradation of PML-RARA oncoprotein and myeloid cell differentiation by enhancing the autophagy pathway. Cell Death Differ 24: 212-224 2016.

41. Yan X, Huang L, Liu L, Qin H and Song Z: Nuclear division cycle 80 promotes malignant progression and predicts clinical outcome in colorectal cancer. Cancer Med 7: 420-432, 2018.

42. Ahonen LJ, Kallio MJ, Daum JR, Bolton M, Manke IA, Yaffe MB, Stukenberg PT and Gorbsky GJ: Polo-like kinase 1 creates the tension-sensing $3 \mathrm{~F} 3 / 2$ phosphoepitope and modulates the association of spindle-checkpoint proteins at kinetochores Curr Biol 15: 1078-1089, 2005.

43. Zhang Z, Zhang G, Gao Z, Li S, Li Z, Bi J, Liu X, Li Z and Kong $C$ : Comprehensive analysis of differentially expressed genes associated with PLK1 in bladder cancer. BMC Cancer 17: 861,2017

44. Yu T, Li J, Yan M, Liu L, Lin H, Zhao F, Sun L, Zhang Y, Cui Y, Zhang F, et al: MicroRNA-193a-3p and -5p suppress the metastasis of human non-small-cell lung cancer by downregulating the ERBB4/PIK3R3/mTOR/S6K2 signaling pathway. Oncogene 34: 413-423, 2015. 
45. Cao G,Dong W,Meng X,Liu H,LiaoH andLiu S:MiR-511 inhibits growth and metastasis of human hepatocellular carcinoma cells by targeting PIK3R3. Tumour Biol 36: 4453-4459, 2015.

46. Klahan S, Wu MS, Hsi E, Huang CC, Hou MF and Chang WC: Computational analysis of mRNA expression profiles identifies the ITG family and PIK3R 3 as crucial genes for regulating triple negative breast cancer cell migration. BioMed Res Int 2014: $536591,2014$.

47. Liu K, Li X, Cao Y, Ge Y, Wang J and Shi B: MiR-132 inhibits cell proliferation, invasion and migration of hepatocellular carcinoma by targeting PIK3R3. Int J Oncol 47: 1585-1593, 2015.

48. Zhu Y, Zhao H, Rao M and Xu S: MicroRNA-365 inhibits proliferation, migration and invasion of glioma by targeting PIK3R3. Oncol Rep 37: 2185-2192, 2017.

49. Wang G, Yang X, Li C, Cao X, Luo X and Hu J: PIK3R3 induces epithelial-to-mesenchymal transition and promotes metastasis in colorectal cancer. Mol Cancer Ther 13: 1837-1847, 2014.

50. Calderón A, Ortiz-Espín A, Iglesias-Fernández R, Carbonero P, Pallardó FV, Sevilla F and Jiménez A: Thioredoxin (Trxo1) interacts with proliferating cell nuclear antigen (PCNA) and its overexpression affects the growth of tobacco cell culture. Redox Biol 11: 688-700, 2017.

51. Yang Q, Ou C, Liu M, Xiao W, Wen $C$ and Sun F: NRAGE promotes cell proliferation by stabilizing PCNA in a ubiquitin-proteasome pathway in esophageal carcinomas. Carcinogenesis 35: 1643-1651, 2014.

52. Kadariya Y, Cheung M, Xu J, Pei J, Sementino E, Menges CW, Cai KQ, Rauscher FJ, Klein-Szanto AJ and Testa JR: Bap1 Is a bona fide tumor suppressor: Genetic evidence from mouse models carrying heterozygous germline Bapl mutations. Cancer Res 76: 2836-2844, 2016.

53. Dey A, Seshasayee D, Noubade R, French DM, Liu J, Chaurushiya MS, Kirkpatrick DS, Pham VC, Lill JR, Bakalarski CE, et al: Loss of the tumor suppressor BAP1 causes myeloid transformation. Science 337: 1541-1546, 2012.

54. Field MG, Durante MA, Anbunathan H, Cai LZ, Decatur CL, Bowcock AM, Kurtenbach S and Harbour JW: Punctuated evolution of canonical genomic aberrations in uveal melanoma. Nat Commun 9: 116, 2018.

55. Smit KN, van Poppelen NM, Vaarwater J, Verdijk R, van Marion R, Kalirai H, Coupland SE, Thornton S, Farquhar N, Dubbink HJ, et al: Combined mutation and copy-number variation detection by targeted next-generation sequencing in uveal melanoma. Mod Pathol 31: 763-771, 2018.

56. Pitcovski J, Shahar E, Aizenshtein E and Gorodetsky R: Melanoma antigens and related immunological markers. Crit Rev Oncol Hematol 115: 36-49, 2017.

57. Garfield EM, Walton KE, Quan VL, VandenBoom T, Zhang B, Kong BY, Isales MC, Panah E, Kim G and Gerami P: Histomorphologic spectrum of germline-related and sporadic BAP1-inactivated melanocytic tumors. J Am Acad Dermatol 79: 525-534, 2018
58. Tetzlaff MT, Torres-Cabala CA, Pattanaprichakul P, Rapini RP, Prieto VG and Curry JL: Emerging clinical applications of selected biomarkers in melanoma. Clin Cosmet Investig Dermatol 8: 35-46, 2015.

59. Balasubramani A, Larjo A, Bassein JA, Chang X, Hastie RB, Togher SM, Lähdesmäki H and Rao A: Cancer-associated ASXL1 mutations may act as gain-of-function mutations of the ASXL1-BAP1 complex. Nat Commun 6: 7307, 2015.

60. Scotto L, Narayan G, Nandula SV, Arias-Pulido H, Subramaniyam S, Schneider A, Kaufmann AM, Wright JD, Pothuri B, Mansukhani M and Murty VV: Identification of copy number gain and overexpressed genes on chromosome arm $20 \mathrm{q}$ by an integrative genomic approach in cervical cancer: Potential role in progression. Genes Chromosomes Cancer 47: 755-765, 2008.

61. Katoh M: Functional and cancer genomics of ASXL family members. Br J Cancer 109: 299-306, 2013.

62. Katoh M: Functional proteomics of the epigenetic regulators ASXL1, ASXL2 and ASXL3: A convergence of proteomics and epigenetics for translational medicine. Expert Rev Proteomics 12: 317-328, 2015.

63. Sahlberg KK, Hongisto V, Edgren H, Mäkelä R, Hellström K, Due EU, Moen Vollan HK, Sahlberg N, Wolf $M$, Børresen-Dale AL, et al: The HER2 amplicon includes several genes required for the growth and survival of HER2 positive breast cancer cells. Mol Oncol 7: 392-401, 2013.

64. Patel VN, Gokulrangan G, Chowdhury SA, Chen Y, Sloan AE, Koyutürk M, Barnholtz-Sloan J and Chance MR: Network signatures of survival in glioblastoma multiforme. PLOS Comput Biol 9: e1003237, 2013.

65. Deng S, Zhou H, Xiong R, Lu Y, Yan D, Xing T, Dong L, Tang E and Yang H: Over-expression of genes and proteins of ubiquitin specific peptidases (USPs) and proteasome subunits (PSs) in breast cancer tissue observed by the methods of RFDD-PCR and proteomics. Breast Cancer Res Treat 104: 21-30, 2007.

66. Qi T, Zhang W, Luan Y, Kong F, Xu D, Cheng G and Wang Y: Proteomic profiling identified multiple short-lived members of the central proteome as the direct targets of the addicted oncogenes in cancer cells. Mol Cell Proteomics 13: 49-62, 2014.

This work is licensed under a Creative Commons Attribution-NonCommercial-NoDerivatives 4.0 International (CC BY-NC-ND 4.0) License. 